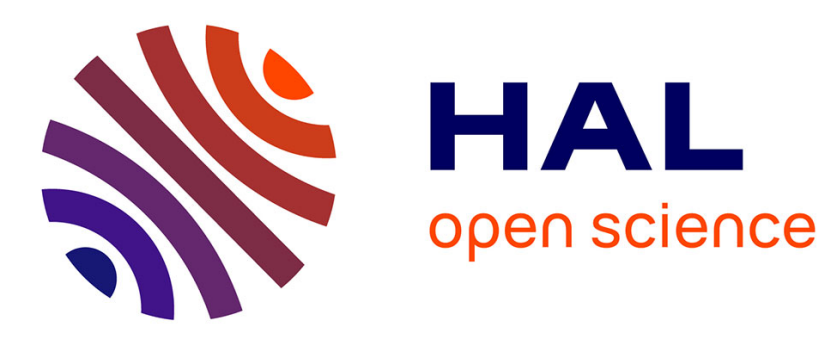

\title{
La diffusion du silex des Costières du Gard au Paléolithique supérieur
}

Sophie Grégoire, Frédéric Bazile

\section{To cite this version:}

Sophie Grégoire, Frédéric Bazile. La diffusion du silex des Costières du Gard au Paléolithique supérieur. Comptes Rendus. Palevol, 2005, 2005 (4), pp.413-419. halshs-00077782

\section{HAL Id: halshs-00077782 \\ https://shs.hal.science/halshs-00077782}

Submitted on 4 Jun 2006

HAL is a multi-disciplinary open access archive for the deposit and dissemination of scientific research documents, whether they are published or not. The documents may come from teaching and research institutions in France or abroad, or from public or private research centers.
L'archive ouverte pluridisciplinaire HAL, est destinée au dépôt et à la diffusion de documents scientifiques de niveau recherche, publiés ou non, émanant des établissements d'enseignement et de recherche français ou étrangers, des laboratoires publics ou privés. 


\title{
PALEONTOLOGIE HUMAINE ET PREHISTOIRE Archéologie préhistorique
}

\section{La diffusion du silex des Costières du Gard au Paléolithique supérieur.}

\author{
Sophie Grégoire \\ Centre Européen de Recherches Préhistoriques, Département de Préhistoire du Muséum National d’Histoire \\ Naturelle, Avenue Léon-Jean Grégory, 66720 Tautavel, France \\ Frédéric Bazile \\ Equipe « Archéologie et Ecologie de l’Anthropisation, UMR 5059 CNRS/Université de Montpellier II/EPHE, \\ Institut de Botanique, 163, rue A. Broussonet, 34090 Montpellier, France
}

\begin{abstract}
Résumé -
Les auteurs soulignent l'importance du Silex en galet des Costières de Gard durant le Pléistocène supérieur et plus particulièrement le Paléolithique supérieur.

Au Paléolithique et surtout au Magdalénien, « l'exportation » des matériaux « Costières », est reconnu largement au-delà des 200 km des gîtes supposés ou actuellement accessibles.

Il ressort de cette première analyse un courant original, fort, Est -Ouest, sans doute encore sous-estimé. Sauf, peut être, pour les Piles Loins (Vauvert Gard), un courant réciproque n’est pas encore établi.

En l'état actuel des travaux, le silex des Costières semble avoir été diffusé au Magdalénien jusque dans les zones de piémont pyrénéen (Belvis et les Conques). La question est de savoir si ce matériau a franchi la barrière pyrénéenne avec les hommes ou si les Pyrénées ont constitué à cette époque un obstacle comme elles l’ont été pour certaines espèces animales comme le renne.

Si l’on admet une certaine rareté du silex de type «Costières » à l'est du Rhône (Crau), ce dernier pourrait se révéler un excellent marqueur de contacts entre Languedoc et Provence, malgré le rôle supposé et admis du Rhône comme barrière culturelle après le Gravettien.

Les auteurs envisagent une étude approfondie du silex «Costières » sur tous ses aspects, diffusion et en particulier vers le nord (Ardèche), vers l'est et au sud des Pyrénées, mais aussi une meilleure caractérisation y compris l'identification des sources primaires potentielles.
\end{abstract}

\section{(Mots clé) : matières-premières / silex / Costières du Gard / Paléolithique / diffusion de matériaux / territoires de circulation.}

\begin{abstract}
-
The authors stress the importance of Flint out of pebble of then Costières of Gard during Pleistocène Supérieur and more particularly the Paleolithic Superior. With Paleolithic and especially in Magdalénien, "the export" of raw materials "Costières", is recognized largely beyond the $200 \mathrm{~km}$ of the supposed or currently accessible lodgings. It comes out from this first analyzes an original strong current, Est-West, is undoubtedly still underestimated. Except possibly for the Piles Loins (Vauvert Gard), a reciprocal current is not established. In the current state of work, the flint of the Costières seems to be diffused in Magdalénien into the zones of Pyrenean Piedmont (Belvis and Conques). The question is to know if this material crossed the Pyrenean barrier with the men or if the Pyrenees constituted at that time an obstacle like that were the case for certain species like the reindeer. If we admits certain scarcity of flint of the type "Costières" in the east of the Rhone (Crau), this last could appear an excellent marker of contact between Languedoc and Provence, in spite of the role supposed and admitted of the Rhone like cultural barrier after Gravettien The authors consider a thorough study of flint "Costières" on all its aspects, diffusion and in particular towards north (Ardeche), the east and in the south of the Pyrenees, but also a better characterization including the identification of the potential primary sources
\end{abstract}

(Key words) Raw materials / flint / Costières du Gard / Paleolithic /raw material diffusion/territories of circulation. 


\section{Introduction.}

Situées à quelques kilomètres au sud-est de Nîmes, entre Camargue et Vistrenques, les Costières du Gard (ou de Nîmes) représentent une entité géographique bien individualisée à l'est de la plaine littorale languedocienne. Elles culminent à 145 m d'altitude NGF à Générac, où la «zone des Puechs » semble émerger d'un plateau qui court de Meynes et Beaucaire à Vauvert, à des altitudes voisines de 80 à 30 mètres NGF.

Les Costières ont fait l'objet de nombreux travaux géologiques, dont les plus récents et les plus aboutis sont ceux de J. Barrière et C. Toni dans les années 1970 [1].

Sans entrer dans les détails, les Costières sont constituées de formations du Pliocène terminal, marin à la base (Plaisancien) puis lagunaires et enfin continentales et détritiques dans la partie sommitale (formation de Surville III). Inscrites dans cette séquence ancienne, et de part et d'autre de la ligne des " puechs », plusieurs nappes alluviales étagées témoignent des différents cours du Rhône depuis le Quaternaire ancien. Plus à l'ouest, au-delà du Rhône, les cailloutis duranciens de la Crau d'Arles ou d'Eyguières procèdent d'un système sensiblement contemporain des cailloutis de Surville III, les grands épandages «villafranchien » qui marquent la fin du remblaiement post-pliocène marin. Au-delà du Gardon, les Costières se prolongent jusqu’à la dépression de Pujault par des dépôts à stratigraphie comparable sinon identique, se terminant par une nappe caillouteuse puissante affectée par une forte pédogenèse: Plateau de Signargues, Bois de Clary...

Pour l'essentiel (Costière et Crau) les cailloutis témoignent d'apports principalement duranciens, caractérisés, entre autre, par des galets de calcaires sub-lithographiques, gris clairs, à dessins elliptiques de microfissures calcitiques. Les roches vertes, ne sont pas exceptionnelles, renforçant le caractère durancien des dépôts. Les apports du Rhône, présents (quartzites alpins), restent relativement discrets au sud, plus marqués au nord, au-delà du Gardon (Signargues, Clary). Selon les données pétrographiques disponibles [1] le silex représente environ $3 \%$ des cailloutis non altérés à dominances calcaire (63 à $61 \%$ en haute Costière) ; ce pourcentage est vraisemblablement accru par concentration des éléments siliceux, au sens large du terme (quartzites inclus), en rapport avec la puissante pédogenèse qui affecte les dépôts (au moins $10 \mathrm{~m}$ ).

Ces nappes anciennes, livrent en quantité appréciable des galets de silex, de morphologie ovoïde et de dimensions allant de 25 à 10 centimètres pour une moyenne de 12 à $15 \mathrm{~cm}$.

A l'inverse, les nappes spécifiquement rhodaniennes (terrasses de la zone nord des Costière, Vistrenque et basse vallée du Rhône) sont relativement pauvres en silex, mis à part quelques rares petits blocs de mauvaise qualité et quelques lydiennes plus ou moins propices à la taille.

Le silex de la Costière et des formations assimilées joue donc un rôle important dans le Paléolithique supérieur régional et, sans doute, au-delà du Languedoc Oriental. Sa diffusion débute vraisemblablement dès le Paléolithique moyen (Moustérien).

Sa collecte présente un certain nombre d'avantages qui n’ont pas dû laisser insensibles les populations du Paléolithique.

- Le néocortex d'alluvions, très typique, permet de le reconnaître très facilement, même entier, au sein des quartzites alpins qui représentent l'essentiel des galets siliceux résiduels de ces dépôts plio-pleistocènes.

- Il est souvent en partie fracturé offrant au tailleur un plan de frappe et souvent une arête permettant de démarrer le débitage sans préparation.

- Il est relativement abondant et débarrassé de toute roche encaissante ; en fait il suffit de se baisser pour le ramasser. 


\section{Le silex des Costières du Gard.}

Le silex des Costières se présente sous forme de galets, plus ou moins ovoïdes de dimensions variables de 5 à $25 \mathrm{~cm}$, plus rarement $30 \mathrm{~cm}$ pour leurs plus grandes dimensions, qui restent exceptionnelles. Ce matériel est caractérisé par un néocortex alluvial typique qui permet de le distinguer sans problèmes dans les séries lithiques du Languedoc Roussillon. En Crau, un matériel comparable n'a pas été formellement identifié, au niveau du cortex du moins. Cette identité se trouve renforcée par l'absence ou l'extrême rareté de silex dans les alluvions des fleuves côtiers de cette région, Vidourle, Hérault, Orb, Aude, Agly, Têt et Tech. De même, les affluents languedociens en rive droite du Rhône livrent peu de silex sauf peut être l'Ardèche, reprenant des éléments aplatis de type plaquette, aisément identifiables.

Les seuls silex en galet qu'il soit possible de retrouver au sud de l'Aude sont les silex miocènes brun-gris du bassin de Bages-Sigean, repris par la Berre au niveau de Portel des Corbières (Corbières nord orientales) et déposés environs 6 km en aval, au niveau du Hameau du Lac, au sud de Sigean, à proximité du littoral.

Le silex noir du Trias de la base du Mont-Tauch, également dans les Corbières, est lui repris par le Verdouble un affluent de l'Agly. Toutefois aucun galet de cette matière n'a été reconnu dans l'Agly malgré sa présence de façon anecdotique dans les alluvions anciennes du Verdouble.

Quoi qu'il en soit, ces matières premières, même à l'état de galet, sont aisément identifiables et n'ont aucune similitude avec les galets des Costières.

Le silex des Costières proprement dit témoigne d'une énorme diversité, normale quand on considère l'importance du bassin versant de ce gîte secondaire. Nous avons souligné par ailleurs [4], les dangers d'interprétations erronées sur de prétendus transports (ou trajets) «longue distance » à partir d'un gîte secondaire ou se mêlent des apports variés, principalement Durancien et Rhodanien. Pour ne prendre qu'un exemple, le seul plateau de Signargues $\left(8 \mathrm{Km}^{2}\right)$ a livré une quarantaine de matière différente [8].

En fait l'unité du silex est donnée par le cortex, généralement un cortex siliceux de couleur ocre plus ou moins régulier et dans la plupart des cas assez fin (- de $2 \mathrm{~mm}$ ). Ce néocortex alluvial se caractérise par la présence de nombreuses micro-géodes de quartz sur sa surface, visibles à la loupe binoculaire, et par des formes d'altérations superficielles, plus rarement profondes de type « choc en coup d'ongle».

\begin{tabular}{ll}
\hline & Galets de silex des Costières du Gard \\
\hline Coloration & marron, beige, brun, rouille, gris, opaque à translucide \\
Texture & très fine et homogène \\
Zonations & irrégulières, sous-corticales \\
Patine & blanche partielle souvent rubéfiée \\
Cortex & siliceux composé de micro-géodes de quartz, surface d'altération de galet \\
Inclusions & bioclastes visibles à l'oeil nu, rares \\
Imprégnations & ferrugineuses \\
Morphologie des galets & ovoïde ou subtabulaire \\
\hline
\end{tabular}

Tableau I : Description synthétique des silex des Costières du Gard Table : synthetic description of Costières du Gard flint

Sous ce cortex à l'aspect constant se cachent plusieurs types de silex aux couleurs variées allant du marron au gris très clair et de l'opaque au translucide. La texture, toujours fine et homogène, est un gage de qualité pour ce matériau alluvial malgré les fissurations subies par les galets durant un transport en forte énergie, parfois sur de longues distances. Une zonation sous-corticale parallèle est souvent présente sur de nombreux types et constitue également une caractéristique de ces silex. Les zones de silex exposées à l'air libre revêtent systématiquement 
une patine blanche partielle qui s'installe de manière progressive en envahissant en premier lieu la zone sous corticale. Des patines antérieures, à l'aspect rubéfié, sont présentes sur certains galets. Des éléments figurés, des gastéropodes, sont visibles à l'oeil nu sur certains types.

La morphologie des galets donne des indications sur la nature des accidents siliceux à l'affleurement. Certains galets semblent issus de rognons aux formes irrégulières, d'autres de bancs de 10 à $12 \mathrm{~cm}$ d'épaisseur. Les galets ovoïdes, les plus représentés semblent issus de nodules de silex sub-arrondis, réguliers. (Tabl.1).

\section{Le silex des Costières du Gard, diffusion d'est en ouest.}

Le silex des Costières est exclusif dans les séries du Paléolithique ancien et moyen de la Costière méridionale [2]; ils représente la quasi-totalité de la matière première à Ioton (Beaucaire), gisement moustérien très proche de cette zone d'approvisionnement. Il est présent de façon plus sporadique dans le Moustérien de type Quina des gorges du Gardon à la Balauzière et à l'Esquicho Grapaou, à coté d'une dominance écrasante des silex de Collorgues Aubussargues (90 \% ) [2]. Plus à l'ouest, cette matière première contraste énormément parmi les types de silex classiquement utilisés. Par conséquent, son identification ne nécessite pas de dispositif d'analyse particulier [11].

On le retrouve à la grotte de l'Hortus (Hérault) avec quatre éclats, dont deux corticaux et à la Crouzade (Aude) avec trois éclats et un nucléus soit à plus de 100 kilomètres des Costières. Sa présence est aisément identifiable parmi les silex du jurassique supérieur du bassin de St Martin de Londres, ou les silex lutétiens de la région de Montpellier ou encore parmi des silex tertiaires lacustres du bassin de Bages-Sigean.

Cet inventaire est loin d'être exhaustif et un examen attentif du Moustérien du Languedoc Roussillon dans une optique d'identification de ce matériau apporterait de nouvelles données.

Au Paléolithique supérieur, objet de notre propos, le silex des costières connaît une large diffusion dès l'Aurignacien. Dans les gorges du Gardon cette matière première a été utilisée par les Aurignaciens « 0 » de l'Esquicho Grapaou et surtout de La Laouza, ou 41\% des nucléus proviennent des Costières du Gard contre seulement 25\% du Ludien du bassin de CollorguesAubussargues. On le retrouve dans l'Aurignacien ancien classique de la Salpêtrière et de la Balauzière [3]. Au-delà de l'Orb sa présence est attestée dans l'Aurignacien de Régismont, importé sous la forme de support et de petits blocs, pour l'un d'entre eux débité sur place [9]. Il a été repéré plus à l'ouest dans la série aurignacienne de Bize, par l'un d'entre nous

Au Gravettien, nous le connaissons, peu abondant, à la Salpêtrière et dans la vallée de la Tave. Les informations manquent pour la partie occidentale du Languedoc et le Roussillon en raison de la rareté du Gravettien au-delà de l'Hérault. Paradoxalement il est peu abondant à la Treille, site de plein air récemment fouillé dans la zone nord des Costières [5]. Les chasseurs gravettiens sont arrivés vraisemblablement avec des pièces supports pré débitées, le façonnage sur place est avéré, et/ou des outils déjà finis. Un débitage de la matière première locale (galet) est néanmoins attesté par de rares nucléus de petite taille, à éclats et à lamelles.

Au Solutréen quelques témoins, dont une pointe foliacée, sont présents à la Salpêtrière et on note quelques rares pièces à Oullins, notamment dans le Solutréen Supérieur. Il est par contre abondant dans l'Episolutréen /Salpêtrien ancien de la Salpêtrière ou il ne représente pas moins de 70\% du silex utilisé dans le niveau 6b du Porche Centre. Il est également présent mais plus difficile à quantifier à la Rouvière (Vallon Pt. D’arc - Ardéche) Au Magdalénien, le silex des Costières semble connaître son expansion maximale, avec dès la phase ancienne, une exploitation intensive, et sans doute l'exportation de pièces lamellaires supports, à partir du site des Piles Loins à Vauvert [7]. Ce gisement associe une zone de vie, dénotant une réalité de durée dans l'occupation, à un faciès d'atelier nettement orienté vers la production de lamelles, à partir de petits galets décalottés ou de petits blocs déjà fracturés, selon un schéma nettement standardisé et en vue de l'exportation des supports de première intention. La matière première 
est empruntée au cailloutis de Surville III du plateau de Cheval Blanc, qui domine le site et l'essentiel de la chaîne opératoire est représenté sur le site.

Sans entrer dans le détail, pour $70 \mathrm{~m}^{2}$ fouillés, l'abondance des nucléus ( 80 ) et des cassons (16) et la faible représentation des lamelles retouchées ( $N^{\circ} 84$ à $86=52$ ), suggère soit une retouche différée des supports bruts, soit l'utilisation différée (ailleurs ?) des supports retouchés. Un site comme celui de Mayan, à Nîmes, sans doute peu ou prou contemporain, pourrait illustrer cette notion latente de complémentarité. L'industrie est constituée de quelques burins et surtout d'assez nombreuses lamelles à dos souvent tronquées (lamelles scalènes), d'un type comparable à celles des Piles Loins, y compris le module. L'utilisation du silex " Costières » est marquée par plusieurs objets à cortex caractéristique, même si le débitage n’a pas été pratiqué sur place. Aux Piles Loins le silex "Costières », la matière première locale, reste largement majoritaire mais n'est cependant pas exclusive. En se basant sur les seuls nucléus de la zone fouillée, le silex local représente $81 \%$ de l'effectif pour 11,5\% de silex lacustre allogène dont $5 \%$ de silex du Ludien du bassin de Collorgues, donc une relation septentrionale de $50 \mathrm{~km}$, clairement établie. Les autres matières allochtones restent à déterminer avec plusieurs pistes possibles, le bassin oligocène de Salinelle et sous réserves quelques rares matériaux des bassins oligo-miocènes de Bages-Sigean (silex à charophytes).

Toujours dans la phase ancienne du Magdalénien le silex " Costières » est largement présent à Vers Pont du Gard, à Camparnaud. Sur les mêmes critères qu'aux Piles Loins, il représente $44 \%$ des nucléus, pour seulement $10 \%$ des nucléus du Ludien de Collorgues, $23 \%$ de silex lacustre indéterminé et $8 \%$ du Cénomanien de l’Uzége, matière dont la présence n’est pas exclue à Vauvert. L'éloignement relatif de la source Costières explique à la fois une diminution de son utilisation et, corrélativement, la diversité des sources d’approvisionnement.

Nous n'avons pour l'instant aucune donnée sur le Magdalénien ancien de l'Aude, à Lassac ou l'essentiel du silex provient du Thanétien local [15].

Au Magdalénien moyen, le silex Costières représente plus de $60 \%$ de la matière utilisée à Fontgrasse (Vers Pont du Gard) dans les gorges du Gardon, à coté d'autres sources comme le Ludien et le Cénomanien de l’Uzège et vraisemblablement le Barrémien de Châteauneuf du Pape [6].

Toujours autour du Pont du Gard, dans le domaine d'une source d'approvisionnement, de l'ordre de $15 \mathrm{~km}$ pour les affleurements les plus proches mais pas les plus riches (Meynes) mais il semblerait que l'utilisation de la matière première " Costières » soit beaucoup plus large durant la première partie des temps magdaléniens languedociens. Dans l'arrière pays, on trouve quelques éléments, au cortex caractéristique, dans la vallée de l'Hérault au Bois des Brousses et à la Roque II. Il diffuse également largement vers l'ouest et l'étude récente du site du Crès à Béziers [13] [14], témoigne de son utilisation importante, 20\%, au moins, en se basant sur les seules pièces avec trace de cortex. Un peu plus au sud, nous l'avons identifié dans la série magdalénienne de la Crouzade (niveau $\mathrm{D}$, collection Héléna) où il est représenté par une lame corticale cassée, un éclat de galet épais à dos cortical, deux lames et un fragment de nucléus.

Dans la série du Magdalénien moyen de Canecaude (Villardonnel, Aude), 4 \% des pièces, fabriquées en silex blond miel à patine blanche ou rouille ont été isolées du reste du matériel issu du Thanétien local ; deux hypothèses de provenances avaient été proposées : St Ferréol et les Costières du Gard. La source la plus proche, St Ferréol avait été retenue comme source la plus logique [10]. Depuis, une meilleure connaissance des différents types de silex des Costières, autorise à prendre cette source en considération, même si elle est distante du site de plus de $150 \mathrm{~km}$.

Il est sans doute présent dans le Magdalénien moyen de Gazel (renseignement M. Langlais). M. Langlais [10] indique sa présence possible dans la grotte de Belvis au Magdalénien supérieur, largement au-delà des 200 km des gîtes supposés ou actuellement accessibles.

Plus récemment, nous l'avons identifié dans un petit lot de pièces de la couche C3 (Magdalénien moyen) de la grotte des Conques (Vingrau, Pyrénées-Orientales), classé en silex d'origine indéterminée [12]. Après réexamen de ces pièces il s'avère qu'au moins un gros éclat à plage corticale est attribuable sans conteste à la source "Costières ». Les autres pièces, moins 
caractéristiques car de dimensions réduites et légèrement patinées, pourraient être issues du même galet dont la présence est attestée par l’éclat portant le néo-cortex alluvial.

\section{Conclusions.}

Cet inventaire, loin d'être exhaustif, montre l'importance inattendue des Costières du Gard au sens large en matière d'approvisionnement de matière première en roche dure siliceuse au Paléolithique. Dès le Moustérien ce gîte fait l'objet d'une exploitation qui ne se limite pas au seul contexte local [2].

Les séries moustériennes situées à l'ouest et au sud-ouest des Costières, parfois à plus de 100 $\mathrm{Km}$ comportent des matériaux prélevés dans ces formations et témoignent de circulations de groupes moustériens, autour de la Méditerranée, depuis la basse vallée du Rhône jusqu’au massif des Corbières.

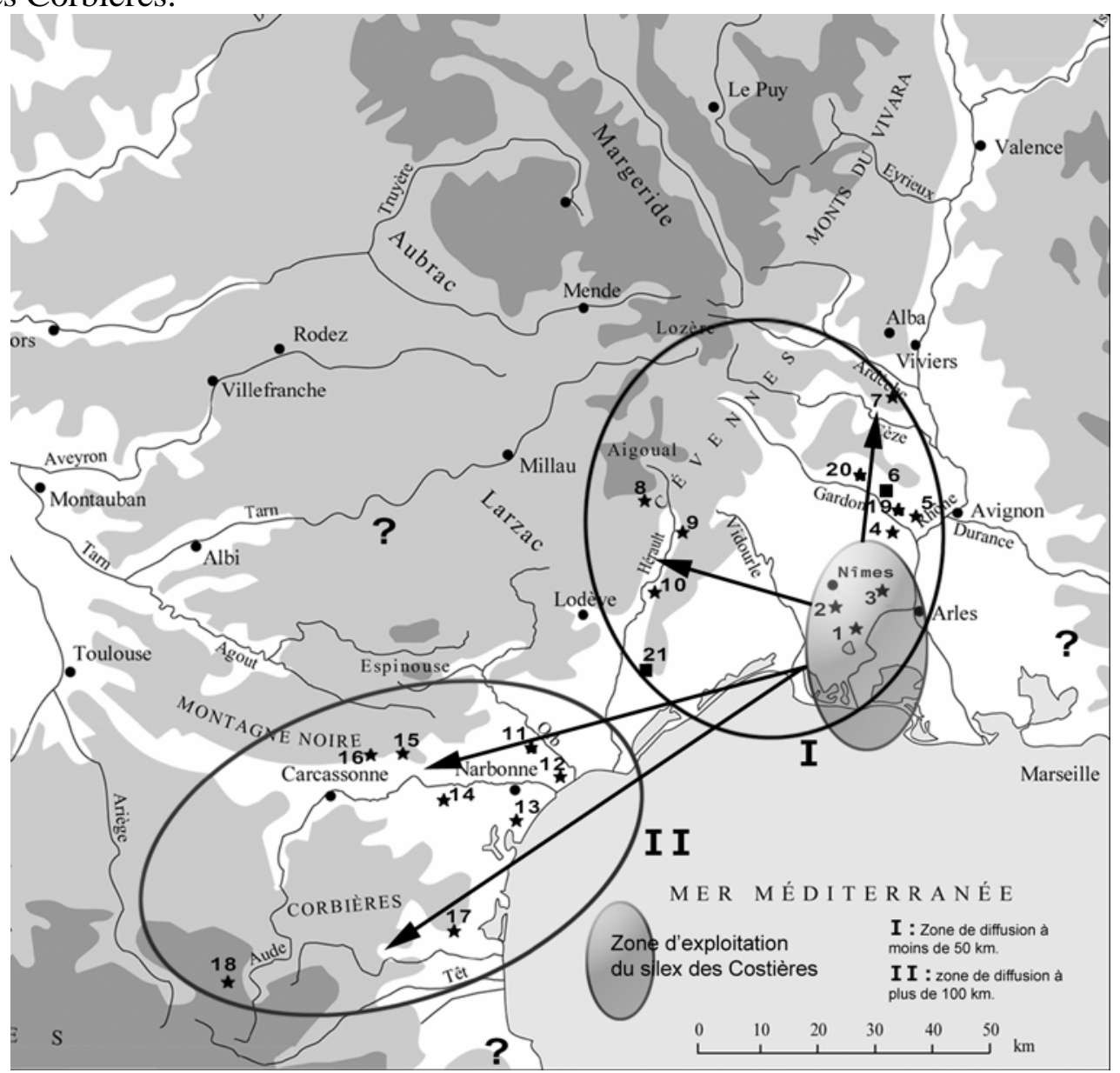

Figure 1 : Diffusion du silex des Costières du Gard Figure 1 : Map of Costières du Gard flint diffusion.

1 : les Piles- loins, 2 : Mayan, 3 : la Treille, 4 : la Salpétrière, 5 : la Laouza, 6 : Camparnaud, 7 : la Rouvière, 8: le Poteau, 9 : La Roque II, 10 : le Bois des brousses, 11 : Régismont, 12 : le Crès, 13 : la Crouzade, 14 : Bize, 15: Lassac, 16 : Canecaude, 17 : les Conques, 18 : Belvis, 19 : Fontgrasse, 20 : la Balauzière, 21 : l'Hortus.

$\mathrm{Au}$ Paléolithique supérieur ce phénomène s'intensifie à l'Aurignacien et surtout au Magdalénien avec « l'exportation » des matériaux « Costières », largement au-delà des 200 km des gîtes supposés ou actuellement accessibles. En effet, le recours à des épandages actuellement immergés au droit du Rhône ne peut totalement être exclu.

Le silex « Costières » est nettement représenté, lorsqu’il n’est pas majoritaire, dans les sites compris dans ce que nous appellerons le territoire principal de diffusion (Fig. 1), zone qui se développe jusqu'à $50 \mathrm{~km}$ au maximum vers l'ouest. Au delà de cette zone, toujours vers l'ouest et le sud-ouest sa représentation diminue plus on s'éloigne de la source dans les sites du 
Paléolithique supérieur mais il est toujours présent et témoigne de circulations sur de longues distances.

Il ressort de cette analyse, très préliminaire, un courant original fort Est -Ouest (Fig. 1), sans doute sous-estimé en l'état des travaux ne prenant en compte que le seul matériel caractéristique, à savoir les artéfacts conservant des plages du néocortex typique des silex des Costières. En l'état, sauf peut être pour les Piles Loins, un courant réciproque n'est pas encore établi.

Ce «sens de circulation » apparaît cohérent pour l'Aurignacien dont l'expansion s'est faite, logiquement ou traditionnellement, d'est en ouest. Elle est plus inattendue pour le Magdalénien ou le sens logique serait plutôt contraire, d'ouest en est si l'on se réfère aux hypothèses les plus communément admises [3] [15]. Indirectement elle pose la question de l'identité du groupe magdalénien moyen de type Fontgrasse et surtout de ses relations avec les groupes plus classiques du Languedoc Occidental.

En l'état actuel de nos travaux, le silex des Costières semble avoir été diffusé au Magdalénien jusque dans les zones de piémont pyrénéen (Belvis et les Conques). La question est de savoir si ce matériau a franchi la barrière pyrénéenne avec les hommes ou si les Pyrénées ont constitué à cette époque un obstacle comme elles l'ont été pour certaines espèces animales comme le renne.

L'identification d'un lien entre le nord et le sud des Pyrénées, déjà établi sur la base de circulation de matériaux [10] encourage la recherche du processus inverse.

Si l'on admet une certaine rareté du silex de type "Costières » à l'est du Rhône (Crau), ce dernier pourrait se révéler un excellent marqueur de contacts entre Languedoc et Provence, malgré le rôle supposé et admis du Rhône comme barrière culturelle après le Gravettien.

A la vue de ces résultats prometteurs, les auteurs se proposent d'inscrire ces problématiques à leur programme de recherche et envisagent une étude approfondie du silex "Costières » sur tous ses aspects. Une meilleure caractérisation par microscopie et géochimie ainsi que l'identification des sources primaires potentielles permettraient de mieux mesurer sa diffusion, en particulier vers le nord (Ardèche), vers l'est (Provence) et au sud des Pyrénées.

\section{Références Bibliographiques}

[1] Barrière J. et Toni C. - Les Costières du Gard : données nouvelles et interprétation. Bull. Soc. Lang. Géogr. 6, 3, (1972) pp. 231-276.

[2]Bazile F. - Le Paléolithique de la Costière Méridionale dans son contexte Géologique. Thèse de Doctorat d'Université des Sciences Naturelles, U.S.T.L. MONTPELLIER (1976), 209 p.

[3] Bazile F. - Le Paléolithique supérieur en Languedoc Orientat. Le milieu, les hommes. ”, 229 p. Habilitation à diriger les recherches, Université de Perpignan, (1998).

[4] Bazile F. - Matières premières minérales et Paléolithique Supérieur en Languedoc Oriental : une entreprise délicate. "Pierre et Archéologie”, pp. 151-176., Presses Universitaires de Perpignan. (2002).

[5] Bazile F., Boccaccio G. \& Monnet-Bazile C. - La Treille (Manduel, Gard) : un nouveau gisement gravettien en Languedoc Rhodanien. (avec G. Boccaccio et C. Monnet - Bazile) Bull. Soc. Préhist . Franç . 9-3 (2001), pp. 546-550.

[6] Bazile F., Guillerault Ph. et Monnet C. - L'Habitat paléolithique supérieur de plein air de Fontgrasse. Travaux 1983-1987. Gallia Préhistoire, (1989), 31, pp. 65-92.

[7] Bazile F., Boccaccio G. \& Ginetti G., - Les Piles Loins (Vauvert, Gard, France), un site de plein air magdalénien à structures conservées en Languedoc-Rhodanien. Bull. Soc. Préhist . Franç . 99-4, (2002), pp. 815-838. 
[8] Boccaccio G.- Alluvions quaternaires et approvisionnements en matériaux de taille pour les hommes préhistoriques : l'exemple de la région de Remoulins (Gard). Bull. Soc.Et. Sc. Nat. Nîmes, 63, (2001) pp. 112-123,

[9] Bon F. - La question de l'unité technique et économique de l'Aurignacien : Réflexions sur la variabilité des industries lithiques à partir de l'étude de trois sites des Pyrénées française, La Tuto de Camalhot, Régismont le Haut et Brassempouy. Thèse, Université de Paris I (2000), 424 p..

[10] Grégoire S. - Origine des matières premières des industries lithiques du Paléolithique pyrénéen et méditerranéen. Contribution à la connaissance des aires de circulations humaines. Thèse, Perpignan. (2000), 246 p.

[11] Grégoire S. - Apports et limites des nouvelles techniques de la pétroarchéologie préhistorique. $C$. R. Acad. Sci. Paris, Sciences de la Terre et des Planètes, 332 (2001), pp.479-482.

[12] Grégoire S.- Origine des matières premières des industries magdaléniennes de la grotte des Conques. In : (sous la direction de Baills H., Moigne A.M, Grégoire S.), la grotte des Conques à Vingrau (Pyrénées-Orientales), des chasseurs et leur territoire. ERAUL 101 (2003) Liège.

[13] Langlais M. - Réflexion sur la place des productions lamellaires au sein de la culture magdalénienne du Languedoc méditerranéen et pyrénéen de l'est. Etude typo technologique de quatte assemblage leptolithique / Montlleo (Prats i Sansor), Le Crès (Béziers, Hérault), Belvis, Aude) et les Piles Loins (Vauvert, Gard). D.E.A., Toulouse-Le-Mirail, (2003), 103 p.

[14] Langlais M. - Les lamelles à dos magdaléniennes du Crès (Béziers, Hérault) : variabilité des modalités opératoires et stabilité typométrique. Bull. Préhist. Sud Ouest, 11-1, (2004) pp. 23- 38.

[15] Sacchi D. - Le Paléolithique Supérieur du Languedoc occidental et du Roussillon. Gallia Préhistoire, XXIe sup., 284 p. (1986), C.N.R.S Paris. 